\title{
Identification of multiresistant Staphylococcus epidermidis in neonates of a secondary care hospital using pulsed field gel electrophoresis and quantitative antibiogram typing
}

\author{
J H Sloos, A M Horrevorts, C P A Van Boven, L Dijkshoorn
}

\begin{abstract}
Aims-To determine the diversity of types of Staphylococcus epidermidis in a neonatal care unit of a secondary care hospital in the Netherlands.

Methods-In a prospective study, specimens from nose, ear, axilla, umbilicus, and groin were taken from patients twice a week during a period of up to two weeks. All isolates were typed by both pulsed field gel electrophoresis (PFGE) and antibiogram analysis.

Results-Fifty three $S$ epidermidis isolates from 15 of 24 patients were obtained in one to four surveys. Fourteen isolates from six patients had a common PFGE pattern and were of one multiresistant antibiogram type. The remaining 39 isolates were allocated to 24 sporadic PFGE types and were more susceptible to antibiotics. Colonisation with the multiresistant strain correlated with a long period of stay and with the use of specific antibiotics. The multiresistant isolates were related closely to isolates of $S$ epidermidis found in a recent study in a teaching hospital in the vicinity of the secondary care hospital.

Conclusion-Repeated sampling and the use of two typing methods allowed the identification of two closely related multiresistant $S$ epidermidis strains in two hospitals in the same area.

(f Clin Pathol 1998;51:62-67)
\end{abstract}

Keywords: Staphylococcus epidermidis; typing; pulsed field gel electrophoresis; antibiogram; antibiotic resistance; neonates

Leiden University

Medical Center,

Leiden, Netherlands

$\mathrm{J}$ H Sloos

C P A Van Boven

L Dijkshoorn

Red Cross/Juliana Children's Hospital, The Hague,

Netherlands

A $M$ Horrevorts

Correspondence to: Dr Sloos, Department of Medical Microbiology L4-P, Leiden University Medical

Center, PO Box 9600, 2300 RC Leiden,

The Netherlands.

Accepted for publication 11 November 1997 that certain strains can persist in neonatal care units. ${ }^{67910}$ In these studies, the spread of the organisms was established by characterisation of isolates on the basis of antibiotic susceptibility spectrum, biotype, plasmid type, and pulsed field gel electrophoresis (PFGE) pattern. At present, a generally accepted typing scheme for $S$ epidermidis does not exist, but PFGE is becoming the standard method for comparative typing of many microorganisms, including Staphylococcus aureus, because of its high degree of reproducibility and its discriminatory power. ${ }^{11}$

In the present study, the prevalence of different strains of $S$ epidermidis was investigated prospectively in the neonatal care units of a secondary care hospital in the Netherlands. Samples were taken consecutively from patients and isolates obtained were typed using PFGE and quantitative antibiogram analysis. The isolates obtained were compared with a predominant strain from a neonatal care unit of a university hospital used in a previous study. ${ }^{10}$

Materials and methods

The study was performed at the neonatal care unit of the Red Cross/Juliana Children's Hospital, The Hague (hospital 1); a secondary care hospital. Most patients are either referred to the hospital by general practitioners or are transferred from obstetric departments of other hospitals in the vicinity of The Hague. The ward has 14 incubators, five of which have a mechanical ventilation facility. Usually, each neonate has its own nurse.

PATIENTS, SAMPLES, AND IDENTIFICATION OF ISOLATES

From 25 October to 22 December 1993, all consecutively admitted patients in the neonatal unit of the hospital were included in the study. Hospital charts and clinical characteristics of patients were evaluated prospectively during the period of study. Swabs from the nose, external auditory canal, axilla, groin, and umbilicus were taken from each patient on Monday and Thursday during a period of up to two weeks, depending on the duration of stay. For the present study, a survey is defined as the sampling of five body sites of one patient on the same day.

Swabs were inoculated on to blood agar (CM271; Oxoid, Basingstoke Hampshire, UK) enriched with $5 \%$ sheep's blood and incubated for 48 hours at $35^{\circ} \mathrm{C}$. On inspection of the plates, a representative colony of each morphotype was isolated. Isolates were identified as $S$ epidermidis if they were: Gram positive cocci, catalase and phosphatase positive, coagulase 
and oxidase negative, susceptible to furazolidone and novobiocin, and producing acid from maltose and sucrose but not from trehalose. ${ }^{412}$

Fifty eight $S$ epidermidis isolates obtained from the neonatal care unit of the Leiden University Medical Center (hospital 2), which is in the vicinity of hospital 1, were also included. These isolates belonged to a common plasmid pattern and were multiresistant to antibiotics. ${ }^{10}$

PULSED FIELD GEL ELECTROPHORESIS

DNA was isolated, digested, and visualised using a modification of the method of Schlichting et al..$^{13}$ Briefly, $50 \mu \mathrm{l}$ of $0.5 \mathrm{M}$ EDTA was added to a $5 \mathrm{ml}$ sample of an overnight culture of the isolate in tryptose soya broth (CM129; Oxoid) grown at $35^{\circ} \mathrm{C}$. A $1.5 \mathrm{ml}$ aliquot of the mixture was centrifuged $(3000 \times g)$ for 15 minutes at $4^{\circ} \mathrm{C}$, and the pellet obtained was resuspended in $250 \mu \mathrm{l}$ of buffer $(10 \mathrm{mM}$ Tris $\mathrm{HCl}$, $1 \mathrm{M} \mathrm{NaCl}, \mathrm{pH}$ 7.6). The suspension was heated to $50^{\circ} \mathrm{C}$ and mixed with $250 \mu \mathrm{l}$ of $2 \%$ low melting point agarose (155-17-022; Gibco, Gaithersburg, Maryland, USA) in TE buffer (10 $\mathrm{mM}$ Tris, $1 \mathrm{mM}$ EDTA, $\mathrm{pH} 8.0)$ and applied to the slots of a template. After $30 \mathrm{~min}-$ utes at $4^{\circ} \mathrm{C}$, plugs were transferred to a 24 well tissue culture plate (Greiner, Frickenhausen, Germany) with $350 \mu$ lysis suspension (10 mM Tris $\mathrm{HCl}, 100 \mathrm{mM}$ EDTA, $50 \mathrm{mM} \mathrm{NaCl}$, $5 \mathrm{mg} / \mathrm{ml} \mathrm{Brij} 58$ (Sigma, Zwijndrecht, Netherlands), $5 \mathrm{mg} / \mathrm{ml}$ NaSarcosyl, $2 \mathrm{mg} / \mathrm{ml} \mathrm{Na}$ deoxycholate, $25 \mu \mathrm{g} / \mathrm{ml}$ lysostaphine (L7386; Sigma), $5 \mu \mathrm{g} / \mathrm{ml}$ lysozyme (L6876; Boehringer Mannheim, Mannheim, Germany)). The plates were incubated overnight at $4^{\circ} \mathrm{C}$, with gentle shaking. Subsequently, $350 \mu \mathrm{l}$ proteolysis buffer (0.5 M EDTA, $10 \mathrm{mg} / \mathrm{ml} \mathrm{NaSarcosyl,} 500 \mu \mathrm{g} /$ $\mathrm{ml}$ protease K (25530-031; Gibco)) was added, and proteolysis was performed for 24 hours at $55^{\circ} \mathrm{C}$ with gentle shaking and one change of the protease containing buffer after eight hours. The plugs were washed with TE buffer while shaking gently for one hour at $4^{\circ} \mathrm{C}$. This washing procedure was repeated four times. The agarose plugs were cut into pieces of $1 \times 5 \times 4 \mathrm{~mm}$ and DNA was digested by immersing the plugs in $200 \mu \mathrm{l}$ restriction buffer containing $30 \mathrm{U}$ of SmaI (Gibco). After incubation overnight at $25^{\circ} \mathrm{C}$, with gentle shaking, the plugs were washed with TE buffer and stored in TE buffer at $4^{\circ} \mathrm{C}$ until electrophoresis was performed. The plugs were placed into the slots of a $0.7 \%$ agarose gel, and the slots were sealed with $0.7 \%$ agarose. The agarose gel was placed into the CHEF-DRII (Biorad, Richmond, Virginia, USA) electrophoresis chamber and electrophoresis was performed at $200 \mathrm{~V}$ for 24 hours at $14^{\circ} \mathrm{C}$ in $0.5 \times \mathrm{TE}$ buffer, with pulse times ranging from one to 30 seconds. After running, gels were stained with $0.5 \mu \mathrm{g} / \mathrm{ml}$ ethidium bromide in water for six hours and photographed. On each gel, one or more specimens of digested S epidermidis LUH3088 (a clinical isolate from our collection) was included as a reference. The relative fragment sizes of this reference strain had previously been determined by comparison with fragments of the chromosomes of Saccharomyces cereviseae.
QUANTITATIVE ANTIBIOGRAM DETERMINATION Antibiograms were determined by disk diffusion on iso-sensi agar (CM473; Oxoid). Suspensions of fresh cultures of bacteria in saline were adjusted to $0.5 \mathrm{McF}$ arland and further diluted $1 / 30$. The resulting suspension was applied to agar plates in order to obtain semiconfluent growth, according to the Dutch national standards for susceptibility testing. ${ }^{14}$ A panel of antibiotics comprising agents with different mechanisms of action was selected and included: chloramphenicol $(30 \mu \mathrm{g})$, ciprofloxacin $(5 \mu \mathrm{g})$, clindamycin $(10 \mu \mathrm{g})$, erythromycin $(15 \mu \mathrm{g})$, fusidic acid $(10 \mu \mathrm{g})$, gentamicin $(10 \mu \mathrm{g})$, methicillin $(10 \mu \mathrm{g})$, penicillin $(10 \mu \mathrm{g})$, rifampicin $(5 \mu \mathrm{g})$, tetracycline $(30 \mu \mathrm{g})$, and trimethoprim $(1.25 \mu \mathrm{g})$. Methicillin susceptibility was assayed by disk diffusion on 5\% sheep's blood agar for 48 hours at $30^{\circ} \mathrm{C}$. Disks were obtained from Becton Dickinson (Cockeysville, Maryland, USA), except for methicillin and clindamycin, which were obtained from Oxoid. The actual inhibition zones were used for cluster analysis.

STATISTICAL PROCEDURES

Isolates were analysed on the basis of their antibiogram. For this purpose the unweighted values of the diameters of the inhibition zones were subjected to cluster analysis with the SPSS statistical software package. ${ }^{15}$ Squared euclidian distances were calculated between all possible pairs of isolates and clusters were generated by Ward's method. ${ }^{16}$ The grouping, depicted in a dendrogram, was inspected for correlations with other features, including PFGE types, and epidemiological data. Patient characteristics were analysed using the SPSS statistical software package and were tested by Fisher's exact test when appropriate (for categorial variables) or by the Student's $t$ test (for continuous variables).

\section{Results}

PATIENTS AND CULTURES

During the sampling period of eight weeks, 24 patients were included in the study, and 164 isolates of coagulase negative staphylococci were obtained. Fifty three isolates $(32 \%)$ from 15 patients were identified as $S$ epidermidis. Isolates not identified as $S$ epidermidis (111 in total) were not analysed further. Five of the 15 patients carrying $S$ epidermidis were born after a caesarean section, two after vacuum extraction, and eight were born spontaneously. The gestation period varied from 26 weeks and six days to 41 weeks and three days (median, 36 weeks and two days), the birth weight varied from $1.07 \mathrm{~kg}$ to $5.05 \mathrm{~kg}$ (median, $2.17 \mathrm{~kg}$ ). Seven patients received mechanical ventilation and 12 had an intraveneous catheter during their stay on the ward. Ten patients received antibiotics, nine of whom were treated with the combination amoxycillin-gentamicin, which is the standard empirical treatment for suspected septicaemia on the neonatal unit. These figures did not differ significantly from the figures obtained for the patients from hospital $2 .^{10}$

The total number of $S$ epidermidis isolates per patient during a maximum of four surveys ranged from 0 to 11 . In the first survey (survey 
A

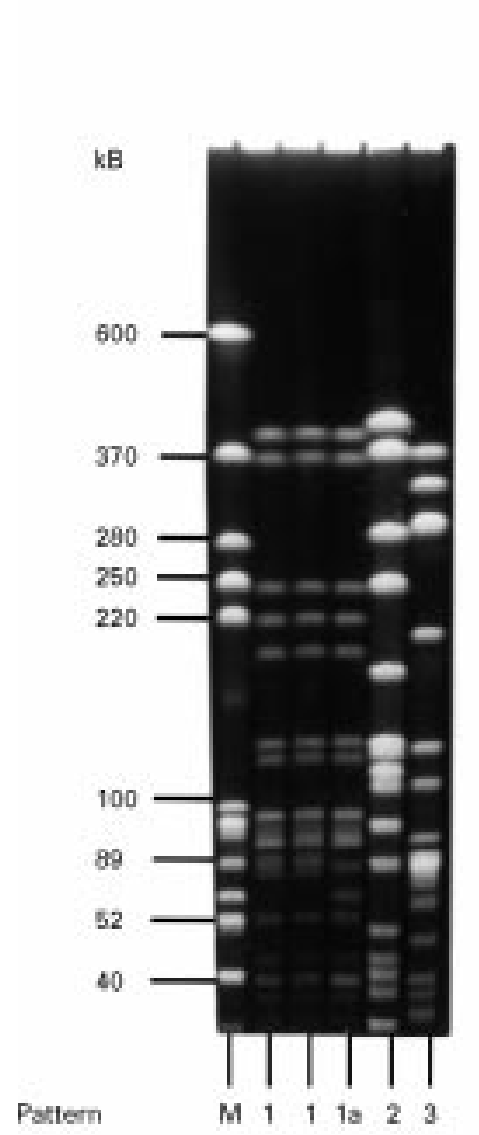

B

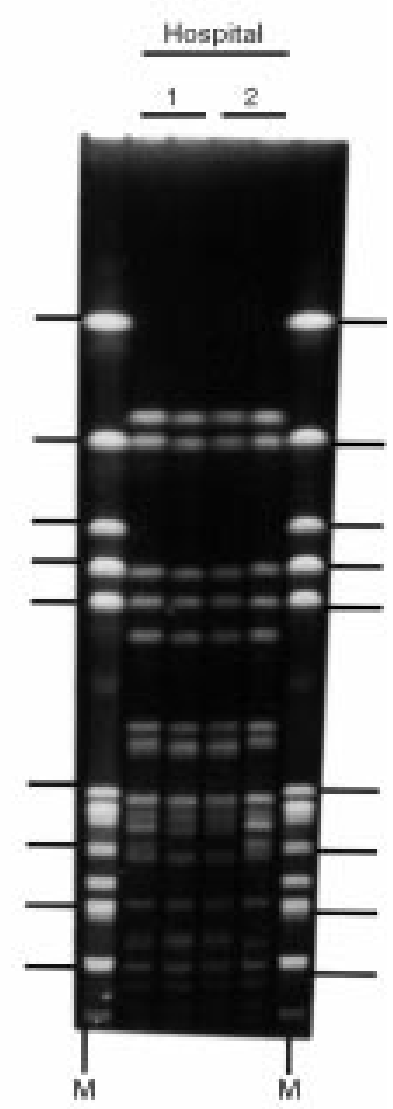

Figure 1 Pulsed field gel electrophoresis patterns of Staphylococcus epidermidis isolates from neonates. Predominant type 1, its subtype 1a, and two sporadic types (types 2 and 3) from hospital 1 (A). Predominant types of hospitals 1 and 2 (B).

1), twenty nine $S$ epidermidis isolates were cultured from 15 neonates. In survey 2, 13 isolates were cultured from six neonates. In surveys 3 and 4, five and six isolates, respectively, were cultured from two neonates. Once $S$ epidermidis was detected in a patient, it was also cultured from this patient in the following surveys. $S$ epidermidis was recovered from all investigated body sites, except for the nose. Twenty two isolates were obtained from the axilla, 17 from the external auditory canal, 12 from the groin, and 2 were from the umbilical cord.

Prospective evaluation of clinical characteristics and review of patient charts did not reveal any clinical infection due to coagulase negative staphylococci during the period of study.

TYPING

Pulsed field gel electrophoresis

The PFGE patterns were characterised by $12-19$ bands ranging from 40 to $600 \mathrm{~kb}$. Types were delineated using the criteria proposed by Tenover et al. ${ }^{17}$ A total number of 25 different types were distinguished within the 53 isolates. In addition, some patterns (subtypes) were found that differed in two or three bands from one of the 25 patterns. These subtypes ${ }^{17}$ were designated by the addition of the letter "a" to their appropriate serial type number. One type (type 1), containing 17 (subtype 1) or 19 (subtype 1a) bands, was observed in 14 isolates from six patients. The remaining 24 types (types 2-25) were sporadic and isolates of these types were obtained from 14 patients. Three sporadic types comprised two subtypes each. The predominant pattern and several sporadic patterns are shown in fig $1 \mathrm{~A}$.

The 58 multiresistant isolates from the neonatal care unit of hospital 2 with a common plasmid pattern (pattern, p1) ${ }^{10}$ were also investigated by PFGE. These 58 isolates had a highly similar PFGE pattern, characterised by 17 or 18 bands (fig 1B). The predominant strains of hospitals 1 and 2 were compared for their PFGE pattern and were found to be closely related according to the Tenover criteria. ${ }^{17}$

\section{Antibiogram typing}

The antibiograms were used for grouping the organisms, but also, the inhibition zones were used for determination of susceptibility categories for each antibiotic according to Dutch standard criteria for susceptibility determination. ${ }^{14}$

The pattern of grouping of isolates on the basis of their antibiograms is shown in the dendrogram of fig 2. The maximum distance between clusters was $59805(100 \%)$. At the delineation level of 18294 (marked by the dotted line), four clusters (A-D) were distinguished. Cluster A contained 20 isolates (10 patients), which were heterogeneous with respect to their antibiograms, as can be derived from their dissimilarity level of 18294 . Cluster B contained four isolates from two patients, linked at a dissimilarity level of 1578, which were resistant exclusively to ciprofloxacin. A third group of isolates (cluster C) comprised 15 isolates (seven patients), clustered at a dissimilarity level of 9019 , and was characterised by relatively large zone sizes. The final group (cluster D) comprised 14 isolates, which clustered at a dissimilarity level of 6758 . Isolates of this group were found in six patients, and were characterised by resistance to six of the 11 antibiotics tested: methicillin, erythromycin, clindamycin, chloramphenicol, gentamicin, and trimethoprim.

Cluster analysis on the basis of antibiograms was also performed on the combined collection of all isolates of hospital 1 and the 58 multiresistant isolates of hospital 2. All 58 isolates of hospital 2 grouped together with the isolates of cluster D at a dissimilarity level of 12997 (results not shown).

The main pattern of grouping by quantitative antibiogram was evaluated for its correlation with other characteristics of the individual isolates (fig 2), including PFGE type, length of stay, time after admission in which isolates of specific types could be cultured, birth weight, mechanical ventilation, usage of antibiotics, and cubicle number. Isolates of cluster $\mathrm{A}$, which were heterogeneous in antibiogram were also heterogeneous in PFGE type. Except for patients with isolates of PFGE type 2 and 6, no neonates were colonised with isolates of the same PFGE type found in cluster A. Isolates of cluster B were from two neonates and were of the same PFGE type. The similarity in PFGE type and antibiogram suggests that these 


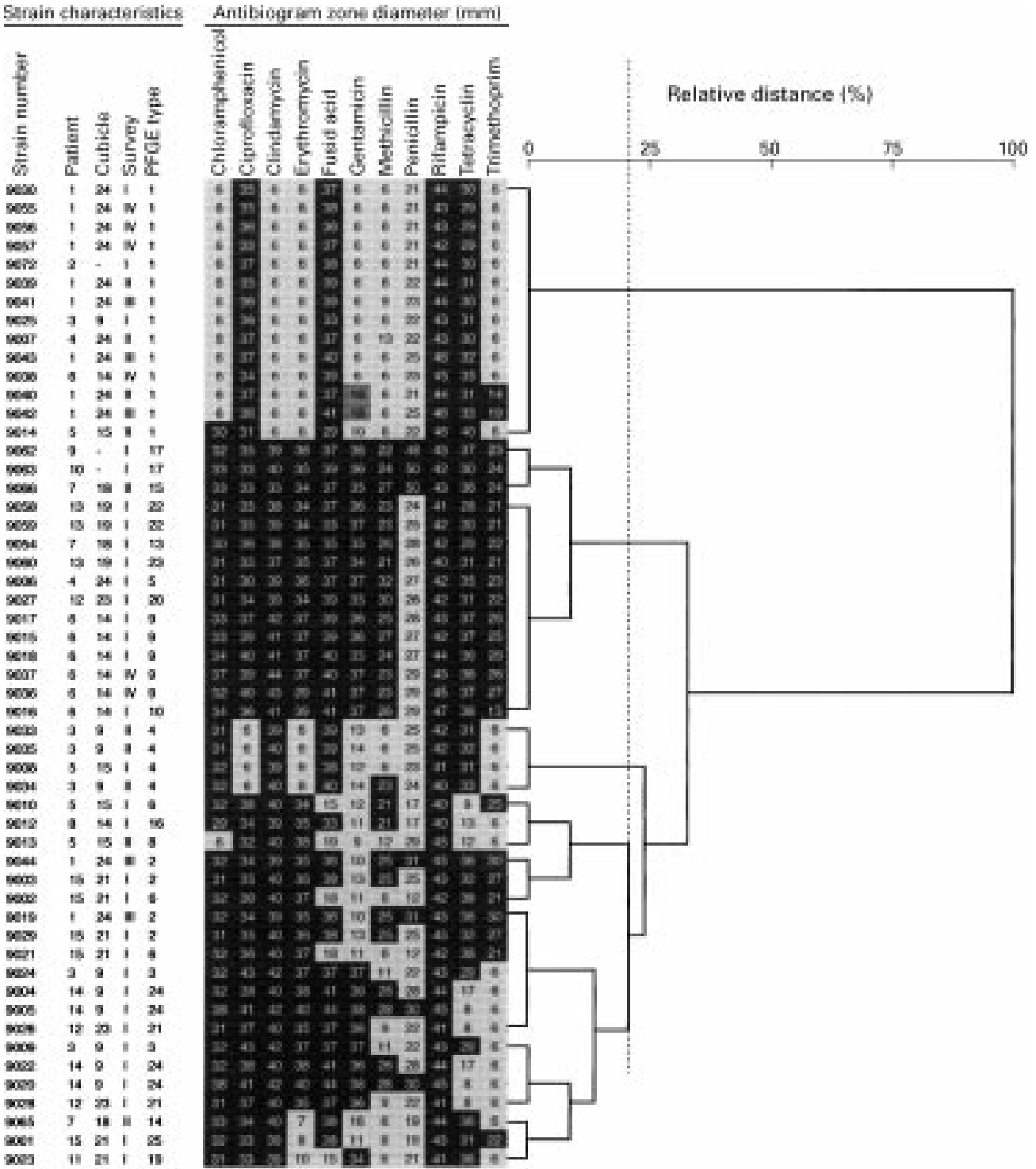

Figure 2 Strain characteristics (left), antibiogram susceptibility profiles (middle), and grouping of 53 Staphylococcus epidermidis isolates of neonates on the basis of zone diameters. Squared Euclidian distance was calculated between all possible pairs of zones and grouping was performed using Ward's method. The dotted line denotes the distance at which clusters A-D are delineated. The inhibition zones were used for classifying isolates into "susceptible" (dark grey), "intermediate resistant" (middle grey), or "resistant" (light grey) categories for each antibiotic, using Dutch standard criteria for susceptibility determination. ${ }^{14}$

neonates carried the same strain. Isolates of the relatively susceptible antibiogram type (cluster C) were cultured from seven neonates. Both of the two isolates of PFGE type 17 were of this antibiogram type and were found in two patients, suggesting that these two patients also carried a common strain. The multiresistant antibiogram type (cluster D) was cultured from six patients and the 14 isolates of this type were all of PFGE type 1 and vice versa. In three of these six patients, the first time that antibiogram type $\mathrm{D}$ was found, was at survey 1 , in two patients it was at survey 2 , and in the other patient it was at survey 4 . The six neonates colonised with the multiresistant isolates of PFGE type 1 were characterised by a mean length of stay of 21.0 days (range, 7-41 days), whereas patients exclusively colonised with isolates of other types had a mean length of stay of 2.9 days (range, $1-6$ days) ( $p<0.001$ ). Isolates of the predominant type were cultured after a mean period of 8.8 days (range, 7-13 days) after admission, whereas isolates of other types were cultured after 4.3 days (range, 1-15 days) $(p=0.095)$. The mean birth weight for both patient groups was $1.97 \mathrm{~kg}$ (range, 1.07$3.72 \mathrm{~kg}$ ) and $2.63 \mathrm{~kg}$ (range $1.49-5.05 \mathrm{~kg}$ ), respectively, but this difference was not significant $(p=0.57)$. Four of six neonates with PFGE type 1 were mechanically ventilated for a mean period of 19 days, whereas three of nine neonates with isolates exclusively of PFGE type 2-23 were mechanically ventilated (mean duration of ventilation, 13 days $)(p=0.179)$. Each of the six neonates with isolates of PFGE type 1 received the combination amoxycillingentamicin, whereas only four out of nine neonates with non-PFGE type 1 isolates received this combination of antibiotics $(\mathrm{p}=0.042)$. Interestingly, neonate 4 who was first admitted into cubicle number 4 carried the strain with PFGE type 1 and antibiogram 
type D. Two weeks later the same cubicle was used for neonate 1 , a patient who also carried the multiresistant isolate of PFGE type 1.

\section{Discussion}

In a previous study the diversity of $S$ epidermidis types in a neonatal care unit of a university hospital (hospital 2) was investigated. ${ }^{10}$ Forty five per cent of the isolates exhibited a common antibiotic susceptibility pattern and plasmid type, and were considered to be one strain. In the present study, the variation or nonvariation of isolates was investigated in a neonatal care unit of a secondary care hospital in the Netherlands. Both the PFGE pattern and antibiogram analysis were used for typing $S$ epidermidis isolates. It was found that 14 of 53 isolates $(26 \%)$, from six of 24 neonates, were of the same PFGE type (type 1) and had a common multiresistant antibiogram. Another group, comprising 15 isolates (28\%) from seven patients, was characterised by a more susceptible antibiotic susceptibility pattern and comprised several PFGE types. The remaining 24 strains were heterogeneous both in antibiogram and PFGE type. The isolates found in this hospital were compared with the predominant strain in hospital 2. On the basis of their highly similar PFGE pattern and antibiogram, the predominant strains in hospitals 1 and 2 were defined as closely related.

The neonates in hospital 1 had shared features that are characteristic of neonatal care unit patients, including low birth weight and the use of both intravascular catheters and antibiotics. ${ }^{12}$ Patient characteristics were evaluated for possible factors influencing the acquisition of the multiresistant strains with PFGE type 1. Comparison of patients with the multiresistant strain of PFGE type 1 and patients not carrying this strain showed that several features of the neonates correlated significantly with the first group of patients. Patients with the multiresistant isolates were characterised by a longer admittance period $(\mathrm{p}<0.001)$, and each of them used the antibiotic combination of amoxycillin-gentamicin $(p=0.042)$. In addition, patients with the multiresistant strain of PFGE type 1 were admitted for a longer period to the neonatal unit before the multiresistant type was isolated. They also had a lower birth weight, and were ventilated mechanically more frequently. However, the latter three differences were not statistically significant. Apparently, the six patients with the multiresistant strain were more seriously ill, and were handled more frequently. Thus, neonates with life threatening conditions seem to be at risk of becoming colonised with multiresistant $S$ epidermidis. Drews et $a l,{ }^{1}$ who performed a prospective surveillance of nosocomial infections in 229 neonates, showed that birth weight was inversely related to the prevalence of infection due to various species. Whether this relation was also true for infection with $S$ epidermidis, the second most frequently isolated causative agent in that study after $S$ aureus, was not mentioned by the authors. In newborn patients in special care units, colonisation with resistant $S$ epidermidis was found to occur after a longer period than colonisation with more susceptible strains, which are acquired within the first few days after birth. ${ }^{18}$ In our study, patients were sampled during successive surveys and in each survey the predominant strain was found in a few patients only. It was by the repeated sampling procedure that its persistence in multiple patients was noticed. Both the antibiogram type and PFGE pattern of these resistant isolates were highly similar, which suggests that the isolates represent a single strain that had spread among patients. The difference in admittance period between the patients colonised with the multiresistant strain (mean, 21 days) and patients with only relatively susceptible strains (mean, three days), and the observation that isolates of the predominant resistant type tended to be cultured after a longer period than susceptible types, suggest that acquisition of the multiresistant strains took place on the ward. It is possible that neonates could have been contaminated via the cubicle, because neonates 1 and 4 used the same cubicle and were both colonised with the multiresistant strain of PFGE type 1. No infections were observed during the period of study and it is not clear whether neonates colonised by resistant strains are at risk of getting infections. The clinical significance of $S$ epidermidis in neonatal care unit neonates is difficult to assess because clear cut criteria for infections in this patient category are missing..$^{19}$ Nevertheless, antibiotics are often prescribed if there is any doubt, which may result in the emergence of antibiotic resistance. Antibiotic usage in the neonatal care unit was high, with 10 of 15 patients colonised with $S$ epidermidis using one or more antibiotic. Each of the six patients colonised with the strain of the multiresistant type was treated with amoxycillin-gentamicin, but it cannot be concluded from our study that the use of antibiotics was a causal factor for the specific acquisition of resistant strains of $S$ epidermidis. Nyström et al showed that differences in antibiotic use were not correlated with colonisation of neonates with specific strains. ${ }^{20}$ Other reports indicate that resistant $S$ epidermidis are associated more commonly with infections ${ }^{679}$ than are more susceptible strains.

In the present study, isolates were typed by the use of PFGE. This method has been proven to be suitable for epidemiological typing of different species, including staphylococci. Similar PFGE patterns are suggestive of clonal relatedness. ${ }^{71}$ The interpretation of patterns can be complicated by the presence of small genetic alterations; these can lead to differences in the banding pattern, with a maximum of three fragments being affected. ${ }^{17}$ Two highly similar PFGE patterns in the isolates of the multiresistant antibiogram type in hospital 1 were observed. The two patterns differed in two bands and were considered to be one type. ${ }^{17}$ Apart from PFGE, antibiogram determination combined with cluster analysis was used as a typing method. Although antibiogram pattern analysis is used frequently for delineating the epidemiological relation of isolates, it is recommended that it be used in combination with other typing methods. ${ }^{21}$ Another frequently used genomic typing 
method for $S$ epidermidis is the determination of plasmid profiles. $^{6918}$ In a previous study performed in a teaching hospital using this method, a predominant multiresistant plasmid type was observed. ${ }^{10}$ Antibiotic resistance genes are frequently located on plasmids, so that similarity in plasmid pattern does not prove clonal relatedness between isolates, but rather may reflect the spread of a plasmid coding for antibiotic resistance. In the present study, 14 isolates of hospital 1 were found to belong to one PFGE type, which corroborates the spread of a multiresistant strain. ${ }^{10}$ Both PFGE and antibiogram analysis gave very similar results for the multiresistant strains from hospitals 1 and 2 . The widespread occurrence of specific strains and clones has been shown for a variety of bacterial species. Further study is needed to clarify the epidemiological significance of the similarity in PFGE types of the multiresistant $S$ epidermidis observed in the two hospitals. In the period of study no patients were transported from one hospital to the other or vice versa. However, exchange of physicians and students between both hospitals occurs. In the neonatal care units in both hospitals the combination of amoxycillin-gentamicin was used as the standard empirical treatment for suspected septicaemia. Possibly, both the spread of strains between the two hospitals and the selection of the multiresistant strain has occurred.

In conclusion, the present study indicates that several neonates acquired a multiresistant $S$ epidermidis strain during their stay in the neonatal care unit of two hospitals. Although no infections were notified, the persistence of such strains in these vulnerable patients should be a cause for concern. Transfer of this strain between hospitals is conceivable and should also be a cause for concern. Detailed studies are needed to assess the clinical significance of multiresistant epidemic strains of $S$ epidermidis in neonates. In these clinical epidemiological studies, typing methods are an indispensable adjunct to more conventional methods and enable precise strain identification and determination of the role of specific strains.

1 Drews MB, Ludwig AC, Leititis JU, et al. Low birth weight and nosocomial infection of neonates in a neonatal care unit. F Hosp Infect 1995;30:65-72.
2 Donowitz LG, Haley CE, Gregory WW, et al. Neonative intensive care unit bacteremia: emergence of gram-positive bacteria as major pathogens. Am F Infect Control 1987;15: $141-7$

3 Jarvis WR. Epidemiology of nosocomial infections in pediatric patients. Pediatr Infect Dis $\mathcal{F}$ 1987;6:344-51.

4 Kloos WE, Bannerman TL. Staphylococcus and micrococcus. In: Murray PR, Baron EJ, Pfaller MA, et al, eds. Manual of clinical microbiology, 6th ed. Washington: American Society for Microbiology, 1991:282-91.

5 Battisti O, Mitchison R, Davies PA. Changing blood culture solates in a referral neonatal intensive care hospital. Arch Dis Child 1981;56:775-8.

6 Carlos CC, Ringertz S, Rylander M, et al. Nosocomial Staphylococcus epidermidis septicemia among very low birth weight neonates in an intensive care unit. 7 Hosp Infect weight neonates in $1991 ; 19: 201-7$.

7 Huebner J, Pier GB, Maslow JN, et al. Endemic nosocomial transmission of Staphylococcus epidermidis bacteremia isolates in a neonatal intensive care unit over 10 years. $\mathcal{F}$ Infect Dis 1994;169:526-31.

8 Nesin M, Projan SJ, Kreiswirth B, et al. Molecular epidemiology of Staphylococcus epidermidis blood isolates from neonatal intensive care unit patients. F Hosp Inf 1995;31: neonatal $111-21$.

9 Lyytikäinen O, Saxen H, Ryhanen R, et al. Persistence of a multiresistant clone of Staphylococcus epidermidis in a neonatal intensive-care unit for a four-year period. Clin Infect Dis 1995;20:24-9.

10 Sloos JH, Dijkshoorn L, Trienekens TAM, et al. Multiresistant Staphylococcus epidermidis in a neonatal care unit. Clin Microbiol Infect 1996;1:44-9.

11 Bannerman TL, Hancock GA, Tenover FC, et al. Pulsedfield gel electrophoresis as a replacement for bacteriophage typing of Staphylococcus aureus. F Clin Microbiol 1995;3: 551-5.

12 Barrow GI, Feltham RKA. Characters of Gram-positive bacteria. In: Barrow GI, Feltham RKA, eds. Cowan and Steel's manual for the identification of medical bacteria, 3rd edn. Cambridge: Cambridge University Press, 1993:50-9. edn. Cambridge: Cambridge University Press, 1993:50-9.
Schlichting C, Branger C, Fournier JM, et al. Typing of Staphylococcus aureus by pulsed field gel electrophoresis, zymotyping, capsular typing, and phage typing: resolution of clonal relationships. $\mathcal{F}$ Clin Microbiol 1993;31:227-32.

14 Mouton RP, van Klingeren B. Standaardisatie van gevoeligheidsbepalingen, Verslag Werkgroep Richtlijnen Gevoeligheidsbepalingen, Bilthoven, The Netherlands: Rijksinstituut voor Volksgezondheid en Milieuhygiëne, 1981.

15 Norusis MJ. Hierarchical clustering. In: SPSS for Windows professional statistics, release 5. Chicago: SPSS Inc 1992:83109.

16 Ward JH. Hierarchical grouping to optimise an objective function. F Am Stat Assoc 1963;58:236-44.

17 Tenover FC, Arbeit RD, Goering RV, et al. Interpreting chromosomal DNA restriction patterns produced by pulsed-field gel electrophoresis: criteria for bacterial strain typing. F Clin Microbiol 1995;9:2233-9.

18 Simpson RA, Spencer AF, Speller DCE, et al. Colonization by gentamycin-resistant Staphylococcus epidermidis in a special care baby unit. 7 Hosp Infect 1986;7:108-20.

19 Garner JS, Jarvis WR, Emori TG, et al. CDC definitions for nosocomial infections. Am f Infect Control 1988;16:128-40.

20 Nyström B, Ransjö U, Ringertz S, et al. Colonization with coagulase-negative staphylococci in two neonatal units. $\mathcal{F}$ Hosp Infect 1992;22:287-98.

21 Blanc DS, Petignat C, Moreillon P, et al. Quantitive antibiogram as a typing method for the prospective epidemiologi-
cal surveillance and control of MRSA: comparison with molecular typing. Infect Control Hosp Epidemiol 1996;17: 654-9 